\title{
Die „Brüste des Nordens“ und Alexanders Mauer gegen Gog und Magog
}

\author{
Andrea B. Schmidt
}

Mauern zum Schutz gegen bedrohliche Völker - in Gestalt etwa des römischen Limes, der chinesischen Mauer oder eines sogenannten „antifaschistischen“ Schutzwalls in der ehemaligen DDR („Antifaschuwa“) - sind geschichtliche Realitäten. Anders verhält es sich mit dem Schutzwall, den Alexander der Große gegen die apokalyptischen Völker Gog und Magog errichtet haben soll. Dieses Bauwerk ist reine Fiktion und in den Bereich der Legende zu verweisen. Gleichwohl hat gerade diese sagenhafte Grenzfeste die Einbildungskraft enorm beflügelt: Alexander soll auf seinen Eroberungszügen die biblischen Völker Gog und Magog ${ }^{1}$ hinter dem Weltengebirge eingeschlossen und so verhindert haben, daß sie vorzeitig, das heißt vor dem Hereinbrechen des apokalyptischen Weltendes, die Erde überfluteten. Die Idee von Alexanders Mauer- oder Torbau ist sowohl in der lateinischen wie in der byzantinischen, christlich-orientalischen und islamischen Kultur entfaltet worden. Wir beschränken uns auf das syrische Milieu, in welchem das Wandermotiv ausgebildet wurde, und fragen nach der Lokalisierung der berühmten apokalyptischen Grenzfeste gegen die wilden Völker.

Die Vermutung liegt nahe, der legendäre Alexanderroman des PseudoKallisthenes aus dem 3. Jahrhundert n. Chr. habe Pate gestanden. Dieser volkstümliche, in einer Unzahl von Versionen verbreitete Roman aus dem spätantiken griechischen Literaturbetrieb hatte die Phantasie in hohem Maße angeregt und war im Mittelalter zu einem beliebten volkstümlichen Text im Westen wie im Osten geworden. Der historische Alexander wird darin zugunsten eines mythischen Helden völlig zurückgedrängt. Auf seine enthistorisierte Person sind im Laufe der verzweigten Überlieferungen des Romans immer

1 Zur unsicheren Etymologie der mythischen Namen vgl. J. Lust, Gog, Magog, in: Dictionary of Deities and Demons in the Bible. Leiden 1995, col. 1000. Zur Identifikation von Gog und Magog mit bestimmten historischen Völkern vgl. das Standardwerk von A.R. Anderson, Alexander's Gate, Gog and Magog and the Inclosed Nations (Monographs of the Medieval Academy of America, 5). Cambridge Mass. 1932, 8-14; W. Brandes, Apokalyptisches in Pergamon, BSl. 48 (1987) 3-4; S. Bøe, Gog and Magog. Ezekiel 38-39 as Pre-Text for Revelation 19,17-21 and 20,7-10 (WUNT, 2. Reihe, 135). Tübingen 2001, 216-218. 
neue phantastische Deutungen projiziert worden. ${ }^{2}$ Der Ursprung des Motivs von Alexanders Schutzwall gegen Gog und Magog stammt indessen nicht aus diesem griechischen Roman, der in seiner ältesten Version $(\alpha)$ das Motiv nicht kennt. Somit ist das Motiv auch nicht enthalten in der im 7. Jahrhundert auf griechischer - und nicht wie lange Zeit angenommen auf mittelpersischer Grundlage entstandenen syrischen Übersetzung ${ }^{3}$ des Alexanderromans. Der für seine phantastischen Abenteuer Alexanders populär gewordene Pseudo-Kallisthenes ist für das Mauermotiv, zumindest was die orientalische Überlieferungstradition angeht, ohne Belang. Das Motiv wird in ausgestalteter Form erstmals greifbar in dem Text der syrischen Alexanderlegende. ${ }^{4}$ Alexander zieht mit einem riesigen Heer aus Alexandrien fort. Nach manchem Abenteuer gelangt er nach Armenien an den Berg Masis. ${ }^{5}$ Er besteigt ihn, besieht sich die Gegend und beschließt, weiter nach Norden vorzudringen bis an das „Tor des großen Gebirges“, eine immense Gebirgskette, die nicht überwunden werden kann. Sie ist von Gott als Grenze zwischen Gog und Magog und der mensch-

2 Vgl. F. Pfister, Kleine Schriften zum Alexanderroman (Beiträge zur klassischen Philologie, 61). Meisenheim am Glan 1976; A.B. Bosworth/E.J. Baynham, Alexander the Great in Fact and Fiction. Oxford 2000.

3 Vgl. C.A. Ciancaglini, Gli natecedenti del Romanzo siriaco di Alessandro, in: R.B. Finazzi/Al. Valvo (eds.), La diffusione dell' eredità classica nell' età tardoantica e medievale. Il „Romanzo di Alessandro“ e altri scritti. Alessandria 1998, 62-68, 72-78, 85, 89-90; Idem, The Syriac Version of the Alexander Romance, Mus 114 (2001) 135, 136, 139.

4 Edition und Übersetzung in E.A.W. Budge, The History of Alexander the Great, Being the Syriac Version. Edited from Five Manuscripts of the Pseudo-Callisthenes, with an English Translation. Accompanied by an Historical Introduction on the Origins and the Various Oriental and European Versions of the Fabulous History of Alexander. Cambridge 1889 (Reprint Amsterdam 1976), 255-275 (Text), 144-158 (Übers.). Zur Frage der jüdisch-hellenistischen Alexandertradition (Josephus etc.) bei der syrischen Ausgestaltung des Torbaumotivs vgl. F. Pfister, Alexander der Grosse in den Offenbarungen der Griechen, Juden, Mohammedaner und Christen. Berlin 1956, 319-327; Anderson, Alexander's Gate (s. Anm. 1), 20.

5 Budge, History of Alexander the Great (s. Anm. 4), 149 übersetzt „der große Musas“. Die Vokalisierung des Konsonantengerüsts $m$-s-s zum Namen „Masis“ ergibt meines Erachtens für Armenien einen besseren Sinn. So auch die Schreibung im syrischen Alexanderlied, vgl. G.J. Reinink, Das syrische Alexanderlied. Die drei Rezensionen (CSCO 455; Script. Syr. 196). Leuven 1983, 38. Mehrere Gelehrte haben einen Zusammenhang sehen wollen zwischen dem Berg Musas bzw. Masis und dem Gebirge Mashu im Gilgameschepos, vgl. K. Czeglédy, The Syriac Legend concerning Alexander the Great, Acta Orientalia Academiae Scientarium Hungaricae 7 (1957) 242, 245; vgl. aber den Einwand von Reinink, Das syrische Alexanderlied, 39 Anm. 64. Moderne türkische Reiseführer beziehen den in der Alexanderlegende genannten Berg auf den Musa Dagh an den Ausläufern des Amanusgebirges, der durch Franz Werfels Roman „Die 40 Tage des Musa Dagh“ über den Widerstandskampf der Armenier im türkischen Genozid 1915 berühmt geworden ist. 
lichen Zivilisation gesetzt worden. Gog und Magog werden als Nachkommen des biblischen Urvaters Japhets bezeichnet. Eingehend beschreibt der Autor der Legende ihr unmenschliches Aussehen und barbarisches Wesen.

Sie haben einen grauenhaften Charakter, schwärmen in Horden aus und beherrschen die Magie. Sie haben blaue Augen, rote Haare und sind in Felle gekleidet. Ihre Frauen sind gleich Amazonen am ganzen Körper mit Messern behängt; sie sind kampfestüchtiger als die Männer und haben nur eine Brust. Diese wilden Völker essen das rohe Fleisch ihrer Toten, sie trinken das Blut von Tier und Mensch. Sie sind schneller als der Wind, und ihre Wagen und Waffen sind wie Blitze. Sie tragen Lassos in den Händen, und jeder von ihnen reitet auf zwei oder drei Pferden zugleich. Die Stimme eines Mannes ist schrecklicher als Löwengebrüll; einer Schar von hundert Mann stehen hunderttausend Truppen Dämonen zur Seite. Sie üben Terror gegen die Menschheit aus.

Der anonyme Autor der Alexanderlegende identifiziert Gog und Magog mit den Hunnen. In seiner übertriebenen Schilderung hat er zweifellos nomadisierende Reitervölker vor Augen. In der Tat hatten die Einfälle von Hunnen und Chazaren vom 4. bis 7. Jahrhundert Kleinasien, Syrien und Mesopotamien wiederholt heimgesucht und sowohl byzantinische Christen wie Perser in Schrecken versetzt. Besonders in einer Szene malt die Legende den Horror dieser unheilvollen Völker aus dem Norden aus. Die Schilderung wiederholt sich ähnlich in der gesamten Alexanderliteratur.

Gog und Magog ergreifen eine schwangere Frau, binden sie vor ein loderndes Feuer und lassen sie braten, bis der Bauch aufbricht und die Leibesfrucht geröstet herausfällt. Dann werfen sie den Embryo in kochendes Wasser, so daß er sich auflöst. Gog und Magog tauchen ihre Waffen in den Sud. Diese Waffenweihe macht sie stark wie hunderttausend Ritter. ${ }^{6}$

Wegen dieser Unreinheit und Wildheit beschließt Alexander, Gog und Magog hinter den Bergen des Nordens einzuschließen. Seine ägyptischen Handwerker bauen ein monumentales Tor (syr. $\operatorname{tar}^{\prime} \bar{a}$ ) aus Eisen und Bronze. Dieses Bauwerk ist in seinen Maßen erstaunlich detailliert beschrieben.

Es ist zwölf Ellen lang und acht Ellen breit. Unten wird eine Schwelle von drei Ellen Höhe in die Berge gehämmert und oben überbrückt ein gewaltiger Türsturz den Abstand zwischen den Bergen. Vier schwere Eisenpfosten, jeder von zwölf Ellen Länge, werden seitwärts um zwei Ellen in die Berge getrieben. Alexander schlägt eigenhändig eiserne Nägel in die gigantische Türschwelle, damit sich Gog und Magog nicht unter dem Tor durchgraben können. Zusätzlich wird das Tor durch eiserne Riegel befestigt und mit einer Schicht aus Eisen und Bronze bedeckt. Allein der Schlüssel für die Schlösser mißt zwölf Ellen. Dann wird das Tor durch eiserne Riegel befestigt und mit einer Schicht aus Eisen und Bronze bedeckt. ${ }^{7}$

6 Budge, History of Alexander the Great (s. Anm. 4), 151.

7 Budge, History of Alexander the Great (s. Anm. 4), 153. 
Die Alexanderlegende entstand um 629 in Nordmesopotamien in der Zeit der großen militärischen Erfolge des Kaisers Herakleios gegen Chosrow II. Die aktuellen politischen Ereignisse werden symbolisch typologisch verarbeitet. Gerrit Reinink bezeichnet die Legende als eine pro-byzantinische Propagandaschrift. ${ }^{8}$ Kernpunkt der Propaganda ist die idealisierte Figur Alexanders des Großen, der als Instrument Gottes Heldentaten vollbringt. Ganz am Anfang des Weltreiches Alexanders steht der Bau des gewaltigen bronzenen Tores, das die apokalyptischen Völker des Nordens, Gog und Magog, abhalten soll. Ihr Einschluß geschieht in Übereinstimmung mit Gottes Heilsplan. Das Tor wird erst am Ende der Weltgeschichte durch ein Zeichen Gottes geöffnet, um mit der Invasion von Gog und Magog die Endzeit einzuleiten. Die syrische Alexanderlegende enthält die älteste Beschreibung des gigantischen Bollwerks Alexanders gegen die apokalyptischen Völker. Das Motiv ist von da an in der westlichen und östlichen Alexanderrezeption - ob griechisch, lateinisch, jüdisch, syrisch oder islamisch - aufgegriffen und stets neu abgewandelt worden. Immer ist von einem gigantischen Bollwerk der Zivilisation die Rede, das Alexander gegen die apokalyptischen Völker erbaut. Von Menschenhand kann es nicht zerstört werden. Erst Gott wird am Ende der Zeiten das Tor öffnen, um Gog und Magog den Weg freizugeben. Die weitere Ausgestaltung des Motivs läßt sich in einem anderen endzeitlich gefärbten Text aus derselben Epoche finden. Das Syrische Alexanderlied ist zwischen 628 und 636 im selben apokalyptisch gefärbten Milieu Nordmesopotamiens entstanden wie die Alexanderlegende und bezieht sich indirekt auf diese. ${ }^{9}$ Die Alexanderlegende war von Kaiser Herakleios' Siegeszügen gegen die Perser bestimmt gewesen. Das Alexanderlied wenige Jahre später steht bereits unter dem Eindruck der ersten arabischen Eroberungen. Die Analyse Reininks zeigt, daß die messianische Rolle Alexanders im Alexanderlied überaus stark betont ist. Alexander agiert von Anbeginn an als Instrument göttlicher Vorsehung, wenn er das Tor gegen Gog und Magog baut. ${ }^{10}$ Er weist seine Schmiede an, wie das Tor gebaut werden soll, und mißt

8 G.J. Reinink, Die Entstehung der syrischen Alexanderlegende als politisch-religiöse Propagandaschrift für Herakleios' Kirchenpolitik, in: C. Laga/J.A. Munitz/L. van Rompay (eds.), After Chalcedon: Studies in Theology and Church History Offered to Albert van Roey (OLA 18). Leuven 1985, 263-282; Idem, Heraclius, the New Alexander. Apocalyptic Prophecies during the Reign of Heraclius, in: G.J. Reinink/ B.H. Stolte (eds.), The Reign of Heraclius (610-641): Crisis and Confrontation. Leuven 2002, 81-94.

9 Reinink, Das syrische Alexanderlied (s. Anm. 5), 12, mit einer Übersicht alternativer leicht abweichender Datierungen. Zur Blüte syrischer apokalyptischer Literatur im 7./ 8. Jahrhundert vgl. Idem, The Beginnings of Syriac Apologetic Literature in Response to Islam, OC 77 (1993) 165-187.

10 Reinink, Die Entstehung der syrischen Alexanderlegende (s. Anm. 8), 281; Idem, Alexander der Grosse und die Lebensquelle im syrischen Alexanderlied, StP 18/4 (1990), 285-287. Anders als in der Legende werden Gog und Magog nicht mit den Hunnen 
eigenhändig den Abstand des Passes zwischen den Bergen. ${ }^{11}$ Das eiserne Tor hat in seinen Ausmaßen noch hinzugewonnen. Der syrische Text spricht von Riesenellen (dganbaruth $\bar{a})$. Nach sechs Monaten ist der gewaltige Bau vollendet. Der anonyme Verfasser des Alexanderlieds schließt:

König Alexander war froh und freute sich sehr über das Bauwerk, wie solide und abwehrfähig und äußerst erschreckend es war; ein Werk, das Weisheit und Intelligenz zustande gebracht hatten. Es zogen Boten aus, die in den Ländern die Nachricht vom gewaltigen Tor verkündeten, das der König gebaut hatte. ${ }^{12}$

Das Alexanderlied wurde in einer krisenhaften Epoche geschrieben. Die Araber haben die Macht in Syrien übernommen. Ein Ausstoß an apokalyptischen Schriften ist die Folge. Sie versuchen, die Ereignisse im Rückgriff auf herkömmliche eschatologische Denkschemata (,septima mundi'-Schema oder das des Propheten Daniel von den vier Weltzeitaltern) geschichtstheologisch zu deuten. So wird im Alexanderlied der Einschluß der biblischen Völker Gog und Magog als ein von Gott vorausgesehenes Ereignis in der Abfolge göttlicher, eschatologischer Entwicklungen gesehen. Während Alexander nach der Legende die Rolle zugefallen war, die heilsgeschichtliche Bedeutung des wiedererstarkten byzantinischen Reiches zu verkünden, wird seine Rolle im Alexanderlied unter dem Eindruck der arabischen Eroberungen eschatologisch interpretiert. Alexander sagt den Zusammenbruch des irdischen Reiches durch Gog und Magog (die Araber) voraus und kündigt das baldige Kommen des himmlischen Königreichs an. ${ }^{13}$

Die bedeutendste apokalyptische Schrift in dieser politisch angespannten Zeit entsteht einige Jahrzehnte später. Auch sie stammt aus Nordmesopotamien. Es ist die Apokalypse des Pseudo-Methodius aus dem ausgehenden 7. Jahrhundert. Auch sie versucht, dem unabwendbaren Vormarsch der Araber und den daraus resultierenden politischen und religiösen Wirren einen Sinn abzuringen. Wie das Alexanderlied ist Pseudo-Methodius beim Torbaumotiv von der Alexanderlegende abhängig, weicht jedoch in einigen Details ab. Eine wichtige Variante ist diese: Im Norden der Welt an den zwei Grenzbergen angekommen ruft Alexander Gott zu Hilfe. Während in der Alexanderlegende und im Lied der $\mathrm{Paß}$ im Gebirge für den Torbau auf natürliche Weise vorgegeben ist, greift in der Methodius-Apokalypse Gott in das Geschehen unmittelbar ein. Gott läßt nämlich die beiden Berge auf Alexanders Gebet bis auf einen zwölf Ellen breiten

identifiziert. Der Verfasser des Alexanderliedes behält die allgemeine biblisch-eschatologische Identifizierung bei, da seine Intention weniger politisch-historisch als theologisch ist.

11 Reinink, Das syrische Alexanderlied (s. Anm. 5), 72-73, 76-77, 92-99 (Rez. II).

12 Reinink, Das syrische Alexanderlied (s. Anm. 5), 97 (Rez. III).

13 Reinink, Die Entstehung der syrischen Alexanderlegende (s. Anm. 8), 281; Idem, Alexander der Große und die Lebensquelle im syrischen Alexanderlied (s. Anm. 10), $285-287$. 
Spalt zusammenrücken. In den Spalt baut Alexander das Tor aus Erz und Eisen, dessen Maße wiederum genau berechnet sind. ${ }^{14}$

Die syrische Apokalypse des Methodius hat sich wie kaum ein anderes literarisches Werk im Osten und im Westen des Römischen Reiches Bahn gebrochen und über den syrischen Kulturkreis hinaus eine außerordentliche literarische Wirkung entfaltet. Bald nach dem syrischen Original sind zwischen 710 und 720 eine griechische und eine lateinische Bearbeitung entstanden. Sie genossen eine außerordentliche Popularität. Hat die syrische Alexanderlegende gewissermaßen die Grundversion von Alexanders Torbau für die syro-orientalischen Traditionen geliefert, so vermittelt die syrische Methodius-Apokalypse jetzt das legendarische Motiv an die griechisch-byzantinische Traditionslinie (Versionen $\beta, \lambda, \varepsilon, \gamma,{ }^{*} \zeta$ ) und an die lateinische Überlieferung des Alexanderromans weiter. Von hier aus entfaltet das Motiv seine universelle Wirkung und dringt sogar in die volkssprachliche Alexanderliteratur des Abendlandes ein. ${ }^{15}$

Die drei genannten syrischen Werke lokalisieren Gog und Magog als Nachkommen Japhets entsprechend dem ersten Buch Moses (1. Mos 10, 2) im Norden der vom Okeanos umspülten bewohnten Erde. Der Norden als dunkle, kalte, unbewohnbare Region gilt als Zentrum des Menschenfeindlichen schlechthin. Für Ełische, den armenischen Historiker aus dem 5. Jahrhundert, war es eine schwere Strafe, nach „Guran und Maguran“ jenseits aller menschlichen Zivilisation verbannt zu werden. ${ }^{16}$ Im Alexanderlied wird diese „rauhe Gegend" als ein Gebirgszug beschrieben, der die ganze Welt umfaßt, eine Grenze, die Gott zwischen die apokalyptischen Völker und die Menschen gesetzt hat. Hier im Kaukasus, der sich nach der Vorstellung der Alexanderlegende bis nach Indien erstreckt und mit dem Hindukusch einen durchgehenden Gebirgszug bildet, lag das Ende der Welt. Die kaukasischen Georgier wurden noch im 19. Jh. von der Karthvelologie als japhetitisches Volk bezeichnet. Die Berge, hinter denen Alexander die barbarischen Völker einschließt, tragen in der griechischen, syrischen und armenischen Überlieferung den Namen „Brüste des

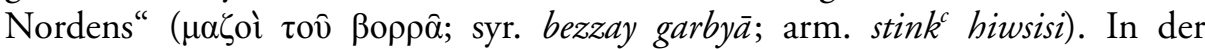
lateinischen Überlieferung der Methodius-Apokalypse und in den mittelalter-

14 G. Reinink, Die syrische Apokalypse des Pseudo-Methodios (CSCO 540,541; Script. Syri 220,221). Leuven 1993, 23-24. Diese Variante des Torbaumotivs ist aus der syrischen Version über die griechische Übersetzung des Pseudo-Methodius in die erweiterte griechische Traditionslinie des Alexanderromans eingedrungen (Rezensionen $\beta, \gamma, \varepsilon, \lambda$ ), vgl. W.J. Aerts/G.A.A Kortekaas, Die Apokalypse des Pseudo-Methodius. Die ältesten griechischen und lateinischen Übersetzungen, I: Einleitung, Texte, Indices Locorum et Nominum; II: Anmerkungen, Wörterverzeichnisse, Indices, (CSCO 569, 570; Subsidia 97, 98). Leuven 1996, 17.

15 Vgl. Pfister, Kleine Schriften zum Alexanderroman (s. Anm. 2)

16 R.W. Thomson, Elishe. History of Vardan and the Armenian War (Harvard Armenian Texts and Studies, 5). Harvard 1982, 208. 
lichen Weltkarten kehrt der Name als „ubera aquilonis“ wieder. ${ }^{17}$ Eine Ausnahme bildet die syrische Methodius-Apokalypse, die die beiden zu einem Pass zusammengerückten Bergen als "Söhne des Nordens“ (syr. bnay garbyā) bezeichnet. ${ }^{18}$ Die unterschiedliche Bezeichnung kommt durch die minimale Verlängerung eines Häkchens bei der Schreibung des syrischen Buchstaben $z$ (bzy) zu $n$ (bny) zustande. Beide Namen sind plausibel. Allerdings ist „Söhne“ in Pseudo-Methodius singulär, während fast die gesamte Tradition von „Brüsten" spricht. Wie wir wissen, hat sich der syrische Verfasser der MethodiusApokalypse bei seiner Darstellung auf eine beliebte Quelle aus dem 5. Jh. gestützt: die syrische Schatzhöhle mit ihrer Nacherzählung der Schöpfungs- und Heilsgeschichte. Der apokryphe Text schildert im Kapitel über den Winter die Geographie des Nordens. Er wird als „Brüste des Nordens“ (bezzai garbayā) bezeichnet, die wie Kristallsteine aussehen. ${ }^{19}$ Die gesamte spätere syrische Tradition wie z. B. Michael Syrus und Bar Hebraeus ist von dieser Tradition der Schatzhöhle abhängig. Auch sie lokalisieren Alexanders Tor bei den „Brüsten des Nordens". Meines Erachtens ist daher dieser Name der ursprüngliche, und der Name „Söhne“ aus einem Schreib- oder Lesefehler entstanden. ${ }^{20}$

Wo genau lokalisieren die syrischen Texte das Tor zwischen den „Brüsten des Nordens"? In der Tat könnte man an den herrlichen Doppelgipfel des Uschba in Svanetien auf der Westseite der Kaukasuskette denken. Manche Texte geben jedoch genauere Hinweise, wo der Torbau Alexanders gegen Gog und Magog zu suchen ist. Danach muß das Bauwerk weiter östlich im zentralen Kaukasusmassiv gesucht werden. Der syrische Patriarch Michael (12. Jh.), der die apokalyptischen Völker mittlerweile mit den Türken identifiziert, präzisiert in seiner Weltgeschichte:

Gog und Magog haben wir selbst gesehen (die Türken) ... Ihr Wohnort erstreckt sich vom Aufgang der Sonne bis zu den Enden des Nordens der bewohnten Erde. Diese Gegend soll von unbezwingbaren Bergen umgeben sein; nur an zwei Stellen gibt es Pforten, durch welche sie hindurchkommen können: eine (Pforte) öffnet sich nach Osten hinein, nach Persien, und die andere nach Norden, ins Innere von Georgien, dort wo sich auch Festungen befinden. Diese letzte ist es, von der man

17 Vgl. Aerts/Kortekaas, Die Apokalypse des Pseudo-Methodius (s. Anm. 14), 115. Im hebräischen mittelalterlichen Alexanderroman werden die Berge als „Promuntorium“ und „Boreum“ bezeichnet, vgl. W.J. van Bekkum, A Hebrew Alexander Romance according to MS London, Jews' College no. 145 (OLA 47). Louvain 1992, 119; Idem, A Hebrew Romance according to MS Héb. 671.5 (B.N.) (Hebrew language and literature series, 1). Groningen 1994, 77.

18 Reinink, Die syrische Apokalypse des Pseudo-Methodios (s. Anm. 14), Kap. VIII, 7.

19 Vgl. A. Su-Min Ri, La Caverne des Trésors, les deux recensions syriaques (CSCO 486; Script. Syr. 207). Louvain 1987.

20 Zu zwei Belegstellen von „Söhnen des Nordens“ in den syrischen Apokalpysen vgl. Reinink, Die syrische Apokalypse des Pseudo-Methodios, II (s. Anm. 14), 23 Anm. 1. 
erzählt, daß sie auf Befehl Alexanders gebaut worden sei, um die Völker von ihrem Auszug abzuhalten. ${ }^{21}$

Michael spricht von den zwei bis in die heutige Zeit strategisch wichtigsten Pässen im Ostkaukasus, der Darialschlucht in Georgien ${ }^{22}$ (auch Ibererwache [griechisch], Schluchtenwache oder Alanentor [armenisch], bzw. Aragwipaß [georgisch]) und dem Derbentpaß in Kaukasisch-Albanien, heute Dagestan (auch Albanertore [griechisch], Kaspische Pforten [syrisch], Hunnenwache bzw. $\mathrm{Pa}$ von Čr [armenisch], Tor der Tore ${ }^{23}$ [arabisch]). Sie erscheinen bei den antiken griechischen und lateinischen Autoren als „Kaukasische Pforten“ oder „Kaspische Pforten“, wobei aus Unkenntnis über die geographischen Gegebenheiten im Kaukasus die Namen in der Regel ohne eindeutige lokale $\mathrm{Zu}$ ordnung verwendet wurden und es zu Verwechselungen zwischen dem Darialund dem Derbentpaß kam. ${ }^{24}$ Die Pässe waren berüchtigt als Durchlässe für kriegerische Völker (Skythen, Alanen, Hunnen, Chazaren, Turkstämme) aus jenen bekanntlich auch heute wieder unruhigen transkaukasischen Gebieten Rußlands. Die Pässe wurden dementsprechend stark von den Römern und Persern befestigt. Besonders der Darialpaß war ein wunder Punkt in der Kaukasusverteidigung. Strabon beschrieb in seiner Geographie den schwierigen Aufstieg über die georgische Heerstraße in die enge, von den hohen Ausläufern des Kasbegs gesäumte Darialschlucht. ${ }^{25}$ Die enge Passage entwickelte sich seit der zweiten Hälfte des 5. Jh. unter den Hunneneinfällen zu einem Dauerproblem. ${ }^{26}$ Der Streit zwischen Römern und Persern um die Instandhaltung der kostspieligen Kaukasusverteidigung und die Tatsache, daß die Römer den persischen Forderungen nur unwillig nachkamen, bildeten einen ständigen Konfliktherd zwischen den politischen Kontrahenten, die im Kaukasus um die Vorherrschaft rangen.

21 J.-B. Chabot, Chronique de Michel le Syrien, patriarche jacobite d'Antioche (11661199), Paris 1899/1901 (Reprint Bruxelles 1963), vol. III, 151.

22 Vgl. L. Dillemann, Haute Mésopotamie orientale et pays adjacents: contributions à la géographie historique de la région du Ve s. avant l'ère chrétienne au VIe $s$. de cette ère. Beirut 1962, 92-93.

23 Vgl. E. Kettenhofen, Art. Darband, in: E. Yarshater (ed.), Encyclopaedia Iranica VII (1996) $13-14$.

24 Vgl. A.R. Anderson, Alexander at the Caspian Gates, in: TAPA 59 (1928) 135-138; J. Kolendo, Sur le nom de Caspiae Portae appliqué aux cols du Caucase, Folia Orientalia 24 (1987) $141-148$.

25 D. Braund, Georgia in Antiquity. A History of Colchis and Transcaucasian Iberia 550 BC-AD 562. Oxford 1994, $46 \mathrm{f}$.

26 Braund, Georgia in Antiquity (s. Anm. 25), 270; A. Luther, Die syrische Chronik des Josua Stylites (Untersuchungen zur antiken Literatur und Geschichte 49). Berlin/New York 1997, 108, 114-115, 136. 
Die syrische Lokalisierung von Alexanders Tor am Darielpaß ist somit eine alte Tradition. ${ }^{27}$ Erst die islamische Rezeption der Alexandertradition hat das Bauwerk auf die Pforte von Derbent übertragen, obgleich diese Lokalisierung mit der syrischen Beschreibung der Mauer Alexanders zwischen einem Paß, der von zwei Bergen gebildet wird, nicht übereinstimmt. Der Derbentpaß liegt nicht zwischen zwei Bergen, wie es die ältere Tradition will, sondern auf einer schmalen, durch einen Bergvorsprung an der west-östlichen Kaukasuskette unterbrochenen Küstenebene, zwischen Gebirge und Kaspischem Meer.

Wie stehen die beiden großen Völker im Kaukasus selbst, die Georgier und die Armenier, zu dieser Überlieferung? Obgleich der Schutzwall gegen Gog und Magog in der syrischen, griechischen und äthiopischen Überlieferung ${ }^{28}$ des Alexandermythos mit dem Kaukasus assoziiert wird, ist er für die Kaukasier selbst, die Armenier und Georgier, völlig unbekannt. Die um den Kaukasus kreisende literarische Tradition bricht ausgerechnet bei ihnen, vor den Toren des realen Kaukasus, ab. Das ist erstaunlich, denn Alexander nimmt bei Armeniern wie Georgiern als Gründer eines Weltreiches einen wichtigen Platz in ihrer Geschichte ein. Die armenischen Bagratiden im 9. Jh. berufen sich auf Alexander als Herrschergestalt. Auf dem Höhepunkt des geeinten georgischen Reiches im 12. Jahrhundert werden die georgische Königin Tamara und König David als „Alexandrini“ (alexandergleich) bezeichnet. Und nicht zuletzt ist bei beiden Völkern eine Version des Alexanderromans im Umlauf.

Die altarmenische Übersetzung des Alexanderromans ist um 480 übersetzt worden, und zwar aus der ältesten Version $(\alpha)$ des griechischen Alexanderromans. ${ }^{29}$ Es wurde bereits erwähnt, daß diese älteste griechische Fassung die Episode vom Einschluß der apokalyptischen Völker noch nicht enthält. Das erklärt, warum die Armenier das Motiv aus dieser literarischen Quelle nicht kennen konnten. Die syrische Alexanderlegende und das Alexanderlied ist hingegen bei den Armeniern nicht rezipiert worden. Wie steht es mit der Methodius-Apokalypse? Der Knotenpunkt in der Überlieferung des Motivs ist,

27 So auch Anderson, Alexander's Gate (s. Anm. 1), 16; idem, Alexander at the Caspian Gates (s. Anm. 24), 139, 141.

28 Das Torbaumotiv hat sich auch in einem äthiopischen Alexanderroman niedergeschlagen (14. Jh.). Zu den Komponenten des Romans vgl. G. Lusini, Origine e significato della presenza di Alessandro Magno nella letteratura etiopica, Rassegna di Studi Etiopici 38 (1994) 95-118. Zu den neun auf koptisch erhaltenen Fragmenten aus dem 7. Jh., die aber das Motiv nicht enthalten, vgl. O. von Lemm, Der Alexanderroman bei den Kopten. Ein Beitrag zur Geschichte der Alexandersage im Orient. St. Petersburg 1903.

29 Der armenische Übersetzer benutzte daneben noch eine Handschrift der jüngeren, erweiterten Rezension $\beta$, vgl. S.M. Lombardi/G. Uluhogian, Due redazioni per il Romanzo d'Alessandro armeno: Tessere di un mosaico perduto, in: R.B. Finazzi/A. Valvo (eds.), La diffusione dell'eredità classica nell' età tardo antica e medievale. Il „, Romanzo d'Alessandro “e altri scritti (L'eredità classica nel mondo orientale, 2). Alessandria 1998, $157-174$. 
wie oben dargelegt, die syrische Methodius-Apokalypse. Erst durch die Übersetzung der syrischen Apokalypse in die griechische und lateinische Sprache ist das Motiv in die jüngeren, erweiterten Fassungen des griechischen und lateinischen Alexanderromans gedrungen. Diese erweiterte jüngere Fassung des Romans - und somit auch das Mauermotiv - lag den Armeniern nicht vor.

Ob der literarisch so wirkungsmächtige Text der Methodius-Apokalypse bei den Armeniern rezipiert worden ist, ist völlig ungeklärt. Es lassen sich keine Spuren in der altarmenischen Literatur finden. Ein längeres Exzerpt aus der Methodius-Apokalypse, allerdings ohne das Motiv, findet sich erst in indirekter Überlieferung im 13. Jh. bei dem armenischen Historiker Stephanos Orbelian. ${ }^{30}$ In der gleichen Epoche ist auch in einer anonymen apokryphen Schrift in verkürzter Form die Episode aus Methodius bekannt. ${ }^{31}$ Es ist jene Stelle, wo Alexander Gott bittet, die beiden Berge mögen zusammenrücken, damit er die eiserne Tür bzw. Mauer dazwischensetzen kann. Dieses isolierte Exzerpt kann jedoch nicht schlüssig beweisen, ob es bereits vor dem 12./13. Jh. eine vollständige armenische Übersetzung der Methodius-Apokalypse gegeben hat und ob sie aus der syrischen oder griechischen Überarbeitung ins Armenische übertragen worden ist. Der literarisch so wirkungsmächtige Text der MethodiusApokalypse scheint bei den Armeniern vor dem ersten Jahrtausend nicht rezipiert worden zu sein, wie gleichfalls nicht die syrische Alexanderlegende und das Alexanderlied. ${ }^{32}$

Eine Übersetzung der Methodius-Apokalypse oder des erweiterten Alexanderromans ins Georgische hat es ebensowenig gegeben. ${ }^{33}$ Gewiß hat die Person Alexanders in der mittelalterlichen historiographischen Tradition der altgeorgischen Annalen (9. Jahrhundert) einen großen Stellenwert besessen, nirgendwo aber gibt es eine Anspielung auf den Torbau Alexanders im Kaukasus. Die Annalen kennen indessen die Variante, daß der dritte georgische König Mirian (Mitte des 2. Jahrhunderts v. Chr.) den Darialpaß durch einen

30 M.F. Brosset, Histoire de la Siounie par Stéphanos Orbélian. St.-Pétersbourg 1864, 8994.

31 M.E. Stone, Armenian Apocrypha Relating to Adam \& Eve. Edited with Introductions, Translations \& Commentary (Studia in Veteris Testamenti Pseudepigrapha, 14). Leiden 1996, 114-134.

32 Ausführlich zur Alexanderrezeption im Kaukasus vgl. E. van Donzel/A. Schmidt, The Quest for Alexander's Wall against Gog and Magog. Early Christian and Islamic Sources, Kapitel 1 (erscheint Leiden 2008).

33 Die heute bekannte mittelgeorgische Übersetzung des Romans datiert aus dem 16./17. Jh. und wurde aus dem Serbischen übersetzt durch Vermittlung der mittelbyzantinischen Rezension $\zeta$, vgl. K. Kekelidze, History of the Old Georgian Literature, II. Tbilisi 1981, 362 (auf georgisch); U. Moennig, Die spätbyzantinische Renzension * $\zeta$ des Alexanderromans (Neograeca Medii Aevi, 6). Köln 1992, 40. 
Wall (georg. simagre) aus zementierten Steinen befestigt habe, um die Chazaren abzuhalten. ${ }^{34}$

Das Motiv vom Mauer- bzw. Torbau Alexanders erscheint in der syrischen Literatur ausschließlich in apokalyptischem Zusammenhang, hervorgerufen durch den Aufschwung, den dieses literarische Genre im 6. und 7. Jh. durch die politischen Umbrüche in Mesopotamien erlebte. Die Vorstellung von Alexander als „Handwerker Gottes“ ist im Rahmen eschatologischer Spekulationen syrischer Christen entwickelt worden. Die armenische und georgische Literatur ist in derselben Epoche kaum eschatologisch und spekulativ ausgerichtet. Literarisch hat man in Armenien und Georgien offenbar anders als in Mesopotamien auf die kurz aufeinanderfolgenden Ereignisse wie Persersturm, Hunneninvasion und arabische Eroberung reagiert. Kein armenischer oder georgischer Geschichtsschreiber kommt auf die Idee, die befestigten Kaukasuspässe, die als eine alltägliche Realität in den Texten häufig erwähnt werden, apokalyptisch zu deuten oder mit einem Bollwerk Alexanders zu verknüpfen. Wenn armenische und georgische Chronisten des 6.-10. Jh. über die Kaukasuspässe und die Völkerschaften jenseits des Gebirges berichten, dann weisen sie sich in der Regel durch differenzierte Kenntnisse über die lokalen und sozialen Verhältnisse der kaukasischen Bergstämme und asiatischen Steppenvölker aus. Für sie ist der Norden nicht zwangsläufig etwas Bedrohliches. Zwar berichten sie von Gefahren und Spannungen mit den „wilden“ Völkern aufgrund von Raubzügen, die jene veranstalten, aber man treibt mit ihnen auch Handel oder schmiedet militärische Allianzen gegen Persien und Byzanz. Nirgends findet sich in der altarmenischen und altgeorgischen Literatur das Bestreben, die Völkerschaften aus dem Norden mit dem Feindbild Gog und Magog zu identifizieren. Durch die Nähe und die alltägliche Berührung mit den vielsprachigen Völkern diesseits und jenseits des Kaukasus hat sich anders als bei den Syrern ein wirklichkeitsgetreueres geographisches und ethnographisches Weltbild der Nordvölker herausgebildet. Für die Kaukasier taugten die Steppenvölker jenseits des Kaukasus nicht als Zerrbild apokalyptischen Schreckens. Armenier und Georgier besaßen über den Kaukasus selbstgeschöpfte geographische Kenntnisse. Das konnte sie eigenständig gegenüber Anschauungen und Wissensgütern machen, die von weiter entfernt lebenden Völkern wie den Syrern nicht mehr geteilt wurden. Sie blieben resistent gegenüber der in der übrigen christlichen Welt zirkulierenden Idee von Alexanders eschatologischem Schutzwall im Kaukasus.

34 M.F. Brosset, Description géographique de la Géorgie par le Tsarévitch Wakhoucht. St.Pétersbourg 1842, 228. 
